\title{
ACERCA DEL DOMINIO DEL CÓDIGO DE LA ESCRITURA EN AMÉRICA LATINA Y SU RELACIÓN CON LOS DESAFÍOS ACTUALES: CONCLUSIONES DE UNA INVESTIGACIÓN EN SIETE PAÍSES DE AMÉRICA LATINA
}

\author{
MARia IsABel Infante*
}

\begin{abstract}
RESUMEN: El artículo presenta la disminución de las tasas de alfabetización en América Latina, mostrando sin embargo que, incluso en los países en que esas tasas son muy reducidas, la población adulta con escolaridad incompleta representa un alto porcentaje. Más adelante, expone algunas conclusiones de la investigación patrocinada por UNESCO/OREALC - que intentaba estudiar el alfabetismo de la población adulta en la capital o ciudades principales de Argentina, Brasil, Colombia, Chile, México, Paraguay y Venezuela, tanto en su fase cuantitativa como cualitativa. Como principal resultado destaca que en esos países, la escolaridad completa no garantiza un verdadero dominio de las competencias de lectura y matemáticas. Igualmente señala la importancia del uso de las habilidades, especialmente en el trabajo, y la relevancia que tienen en los resultados variables como la escolaridad de los padres, la zona de nacimiento, entre otras. Es, al mismo tiempo, una llamada de atención acerca de la importancia de considerar en las reformas educacionales emprendidas por los países a la Educación de Personas Jóvenes y Adultas, especialmente dadas las exigencias que demanda la sociedad de la información y el conocimiento a personas que ya se encuentran insertas en el campo laboral.
\end{abstract}

Palabras claves: Alfabetismo. Investigación. América Latina. Educación de adultos.

Doctora en Filosofía (Dr. Phil.) por la Universidad de Regensburg, Alemania. Coordinadora Nacional de Educación de Adultos en el Ministerio de Educación de Chile. E-mail: infanteisa@mi-mail.cl 


\section{MASTERING THE WRITTEN CODE IN LATIN-AMÉRICA AND ITS RELATIONSHIPS TO THE CURRENT CHALLENGES: CONCLUSIONS OF A SURVEY INVOLVING SEVEN LATIN-AMERICAN COUNTRIES}

ABSTRACT: This article approaches the decreases in illiteracy rates in Latin America indicating that, even in the countries where such rates are lower, the adult population without complete education still represents a very high percentage. It also exposes some conclusions of the research sponsored by UNESCO/OREALC, that focused on the literacy of adult populations in the capital or main cities of Argentina, Brazil, Colombia, Chile, Mexico, Paraguay and Venezuela from both quantitative and qualitative perspectives. The most important result highlighted is that in those countries complete education does not guarantee one masters reading and mathematics skills. The importance of the use of competence, specially at work, and the relevance of such variables as parents' schooling and place of birth, among others, are also stressed. At the same time, the study draws the attention to the importance of considering the Youth and Adult Education in the educational reforms, especially owing to the requirements of our information and knowledge society, under which the adult population is already working.

Key words: Literacy. Research. Latin America. Adult education.

\section{La realidad educativa de las personas adultas en América Latina}

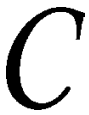

uando se hablaba de la población adulta, durante muchos años la única preocupación de los gobiernos fue el tema del analfabetismo. Se podía afirmar que en el mundo, entre 1980 y 1995 , las tasas de alfabetización de las personas mayores de 15 años habían progresado enormemente (UNESCO, 1995). Sin embargo, en casi todas las subregiones, al mismo tiempo que habían aumentado las tasas, también había aumentado la cantidad absoluta de analfabetos, resultado de que el progreso en alfabetización (o escolarización) había sido inferior al rápido crecimiento de la población.

En el mundo, en el año de 1995, el número de adultos alfabetizados llegó a 1,027 millones y la población creció en 1,035 millones, lo que significa que la población analfabeta creció en 8 millones en esos 15 años.

No así en América Latina y el Caribe en que las personas analfabetas han disminuido en 1 millón: 43 millones en 1995 frente a 44 en 1980.

Aun cuando persiste un mayor analfabetismo femenino en el mundo en general, se puede decir que, en las subregiones en desarrollo, 
la alfabetización de mujeres progresó en forma más rápida que la de los hombres. En general, las distancias disminuyeron en los 15 años. Entre las subregiones, América Latina es la que tiene la menor diferencia entre las tasas de alfabetización de hombres y de mujeres: 4,6 puntos de porcentaje en 1980 y 2,2 en $1995 .^{1}$

Entre los países, la mayor diferencia en cuanto a la alfabetización de hombres y mujeres se da en Bolivia, Guatemala y Perú (entre 10 y 20 puntos). Haití y Guatemala muestran las mayores tasas de analfabetismo entre las mujeres.

La edad promedio de las personas analfabetas en América Latina y el Caribe aumentó de 43 años $(42,8)$ en 1980 a $45(44,8)$ en 1995, lo que muestra que el analfabetismo absoluto está siendo relegado a las personas de mayor edad.

En los grupos de menor edad, especialmente, se perciben los avances de las mujeres en este sentido: las mujeres presentan menores tasas de analfabetismo que los hombres: hasta los 34 años en 1980, situación que se extiende hasta los 39 años en 1995.

Según UNESCO (1995), una vez alcanzada la meta de alfabetización de $70 \%$, el progreso hacia la alfabetización "plena” será sostenido. Sólo quedan, en el año 2000, en América Latina y el Caribe, tres países bajo esa condición: Nicaragua, Guatemala y Haití (UNESCO, 2001).

Brasil, a pesar de tener una alfabetización entre $70 \%$ y $90 \%$, y a pesar de haber disminuido su tasa de analfabetismo de $25,4 \%$ en 1980 a $14,7 \%$ en el año 2000 , presenta, en ese mismo año, 18 millones de personas analfabetas, cuya mayor parte corresponde al sector rural.

No se puede dejar de considerar estos datos a la luz de otras variables, como el índice de pobreza, la mayor cantidad de población rural e indígena. Hay que recordar que los tres países que se encuentran bajo el nivel de $70 \%$ de alfabetización pertenecen al grupo de países clasificados como de alto nivel de fecundidad, urbanización tardía, baja esperanza de vida y bajo nivel económico. ${ }^{2}$

Las proyecciones de UNESCO (1990) de que sólo siete países de América Latina y el Caribe tendrían tasas superiores al 10\% de analfabetismo en el año 2000 resultan ciertas a la luz de los datos actuales, aunque varían algo sus tasas, que son menos positivas que las proyecciones: República Dominicana (16,2\%), Brasil (14,7\%), Bolivia $(14,4 \%)$, Honduras $(27,8 \%)$, El Salvador $(21,3 \%)$, Guatemala $(31,3 \%)$ y Haití $(51,4 \%)$. 
Sin embargo, frente a los avances mostrados por las cifras, habría que recordar, en primer lugar, que estos datos están tomados de los censos de los países, en los cuales sólo se les ha preguntado a las personas si saben leer y escribir. Por tanto, los datos de analfabetismo real pueden diferir un tanto de los números indicados.

Por primera vez (1995) en las cifras de UNESCO se pone énfasis en las tasas de alfabetización ( $\mathrm{y}$ no de analfabetismo), sin embargo, cuando se habla de tasa de "alfabetismo" o "alfabetización", no se sabe de qué calidad es la alfabetización o alfabetismo que la gente dice tener. No sabemos si se trata de "saber firmar", de "saber escribir una carta", de "leer una noticia"...

Las tasas sin duda muestran promedios de los países, tendencias generales, válidas, pero ocultan situaciones concretas, zonas que deben preocupar. La misma UNESCO (1996) subraya la importancia de destacar, por ejemplo, las brechas en las tasas de analfabetismo absoluto de la población indígena de México, Ecuador, Perú, Bolivia y Guatemala, con respecto a los promedios de los países, particularmente en cuanto al analfabetismo femenino.

La calidad de la alfabetización o del alfabetismo impulsa a considerar la relación de las habilidades con los requerimientos del medio en diferentes contextos, es decir, lo que se ha llamado "analfabetismo funcional" o niveles de "alfabetismo".

Los avances de la alfabetización están sin duda relacionados con el progreso de la escolarización en los países de la Región, en las edades entre 6 y 11 años. Este fenómeno, que se observaba ya en la década de 1970 , se ha acentuado favorablemente en las últimas décadas. En la actualidad, más del $90 \%$ de los niños en edad escolar se matricula y el alumno promedio permanece casi siete años en la escuela. Sin embargo existen diferencias importantes entre los países de la región (CEPAL/UNESCO, 1992).

Los países realizan múltiples esfuerzos para disminuir la brecha entre el número de años que los alumnos están matriculados y los grados que han aprobado en la escuela, pero se mantiene un serio problema de calidad, ya que la deserción tiende a comenzar en la mayoría de los países a los 14 años de edad y muchos jóvenes que desertan a esa edad sólo han aprobado unos pocos grados, debido a las altas tasas de repetición (UNESCO, 1996).

Sin embargo, esto no significa que no exista una deserción temprana, en los primeros años de escolaridad básica, que en algunos 
países es considerable. En promedio, alrededor de un $9 \%$ de los alumnos en América Central y del Sur ingresa tardíamente a la escuela o deserta prematuramente de ella (Schiefelbein, 1989).

Aunque entre las causas de una deserción temprana se señalan algunas externas al sistema y provenientes de las condiciones económicas, también aparecen como causa las múltiples repeticiones de los alumnos.

Muchos factores inciden en estos resultados, que no son del caso analizar en este momento, entre los cuales valga señalar la inadecuación de los procesos de enseñanza de la escuela a las estrategias de aprendizaje de los niños de sectores bajos, relacionada con la diferencia de códigos culturales. Este factor, al parecer básico para comprender muchos fracasos, se hace patente en la enseñanza de las matemáticas y del lenguaje, asignaturas que influyen poderosamente en los resultados de los alumnos.

Producto de esta situación y de condiciones todavía más desfavorables del funcionamiento del sistema escolar en las décadas anteriores, un alto porcentaje de la población adulta de América Latina no ha completado su escolaridad básica. Aun en los países en que existe un bajo porcentaje de adultos sin escolaridad (por ejemplo, Argentina, Chile, Uruguay, Costa Rica), el porcentaje de los adultos con escolaridad básica incompleta es, sin embargo, cercana al $40 \%$. En este aspecto hay fuertes diferencias según la zona de residencia (urbana o rural).

Las cifras de cobertura de la población infantil y juvenil mostraban, a comienzos de los años de 1990, que los problemas fundamentales de la educación latinoamericana no residían ya en la cobertura, sino en la calidad y equidad del sistema.

Con la finalidad fundamental de mejorar la calidad y equidad de los sistemas educativos, como también su eficiencia, se emprendieron, en la mayoría de los países de América Latina y el Caribe, profundas reformas que comenzaron con la educación básica para ampliarse posteriormente al resto de los niveles. En ellas se perfilaban cuatro ejes de políticas, en torno a los cuales se diseñan estrategias, programas y proyectos: gestión, equidad y calidad, perfeccionamiento docente, financiamiento (Gajardo, 1999).

Aunque todavía no se logren superar todos los problemas existentes y los avances de la sociedad de la información demanden nuevos enfoques y cambios, no hay duda de que la región se ha movido 


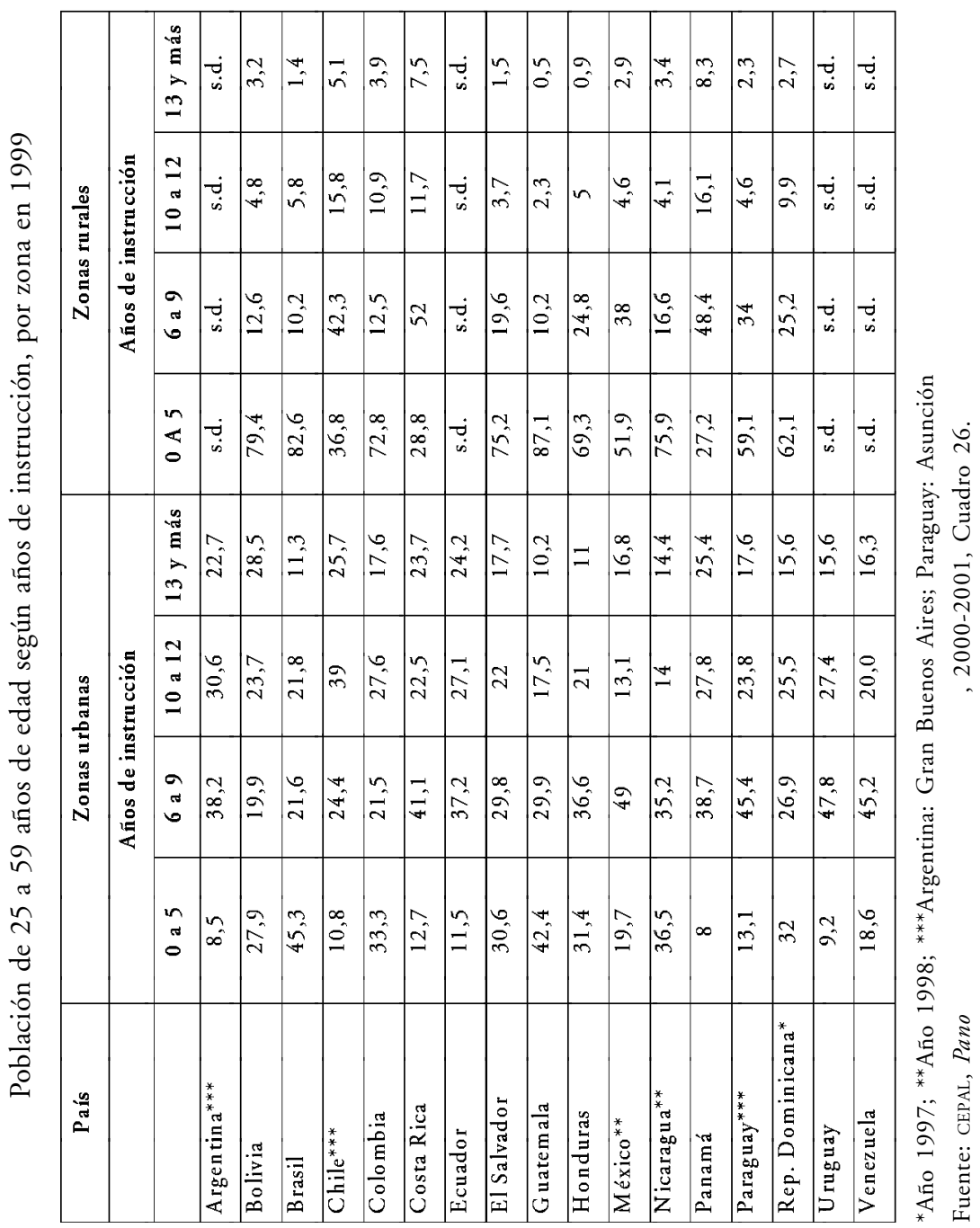


desde una educación tradicional que promovía el acceso a la educación hacia una educación que privilegia los resultados, que focaliza su acción en sectores más pobres, logrando cierta discriminación positiva. Sin embargo, los resultados muestran que los avances no han beneficiado de igual manera a los distintos sectores socioeconómicos.

Esto significa que, a pesar de que los focos hayan sido mejorar la calidad conjuntamente con la equidad, los avances en equidad han sido más reducidos que los primeros. En sociedades tan segmentadas como las latinoamericanas, es lógico pensar que los sectores económicos más altos se beneficien más de las reformas educativas que los sectores más bajos (generalmente de alumnos que asisten a establecimientos municipales o públicos), obteniendo mejores resultados, aun cuando se hayan establecido algunas medidas correctoras de las desigualdades. Las mediciones de resultados así lo demuestran.

En un balance de las reformas latinoamericanas (Gajardo, 1999), se dice que ha habido en la región avances significativos en muchos aspectos, como los acuerdos nacionales en torno a la importancia de la educación y de su reforma como una política de Estado, la mayor descentralización, los programas de mejoría de la calidad y equidad de la enseñanza básica, la puesta en marcha de reformas curriculares, la mayor preocupación por los resultados del proceso de aprendizaje, cierta tendencia positiva hacia la mejoría de la remuneración docente y la profesionalización de su trabajo y una tendencia a una mayor inversión en educación, especialmente en sus niveles inicial y básico.

A pesar de ello, se considera que los resultados son "magros", ya que los países todavía no cuentan con una escuela pública de calidad para todos. Aunque no se han realizado suficientes evaluaciones, surgen explicaciones relacionadas con la lentitud de los cambios en el ámbito educativo, la necesaria relación de la educación con otros factores que producen inequidad social, cuyo efecto la educación por sí sola no es capaz de revertir, obstáculos políticos y técnicos y un financiamiento todavía insuficiente.

Debido a los énfasis de las reformas, promovidos por la discusión de la época y los estímulos y orientaciones del Banco Mundial, hasta el año 2000 no se había enfrentado decididamente en las estrategias de reforma a la población adulta, y especialmente a aquella con escolaridad básica o media incompleta, pese a ser ella la población que trabaja, que debe manejar su vida frente a los acelerados cambios, producto de los poderosos avances en la ciencia 
y tecnología que influyen tanto en el ámbito de su vida privada como en el de su vida laboral y social.

No se había considerado suficientemente que si se quiere mejorar la competitividad internacional a través de la mayor calidad de la producción, introduciendo en el fondo mayor conocimiento y tecnología a los productos, la población adulta debe ser un grupo prioritario.

\section{Las competencias medidas en personas adultas}

Algunas mediciones internacionales de las competencias de lectura y cálculo de las personas adultas han puesto en evidencia la distancia existente entre las competencias de la población adulta en América Latina y las exigencias que imponen la multiplicación de información escrita y la consecuente complejización de la vida diaria, en el contexto de la globalización.

El estudio de la OECD demostró que en Chile (el único país de América Latina que ha participado hasta ahora) más del 50\% de la población adulta había alcanzado sólo el primer nivel - el más bajo de competencias de lectura comprensiva en textos de prosa, de documentos esquemáticos y de cálculo.

Frente a esta medición, sin embargo, es necesario tomar en cuenta algunos aspectos que si bien no invalidan la medición, sin embargo sirven para explicar, por lo menos parcialmente, sus resultados. Esa medición provino de países desarrollados que determinaron ciertos estándares, frente a los cuales otros países, que no tienen el mismo grado de desarrollo, deben medirse.

Sin embargo, hay que pensar que, frente a esos estándares, necesariamente los países en desarrollo y los más alejados de la "cultura" del Primer Mundo llevan un handicap en contra innegable, pues no se puede dejar de plantear la importancia del aspecto cultural en la descodificación de los textos. Numerosos estudios de tipo antropológico han demostrado la influencia de la cultura y del contexto en el desarrollo concreto de estrategias que están presentes en el acto de leer comprensivamente, como están presentes en las mismas estrategias cognitivas (Cole, 1971; Cole y Scribner, 1981). Como el acto de leer es una continua confrontación de hipótesis del lector frente al texto escrito, se movilizan permanentemente estrategias de diferentes tipos: sicolingüísticas, lingüísticas y sociolingüísticas. También juega un papel importante la representación que el lector tiene de la tarea en que está 
inmerso, la que, a su vez, está relacionada con la experiencia de su aprendizaje inicial de la lectura y del significado que ésta tiene para él (Sotomayor, 1995). Todo ello pertenece al ámbito de la cultura y está influido por el estilo cultural de pensamiento de cada grupo social (Infante, 1997).

\section{La investigación regional desarrollada por UNESCO}

Intentando adaptar la medición al contexto latinoamericano, la UNESCO desarrolló, entre 1994 y 1998, una investigación sobre alfabetismo funcional, ${ }^{3}$ que incluía una fase cuantitativa, con instrumentos que seguían el modelo de países desarrollados, pero que incluían textos y situaciones de América Latina, y una fase cualitativa que intentaba descubrir causas más profundas de los resultados y entender mejor la forma cómo las personas con bajos niveles de alfabetismo se relacionan con su ambiente laboral y social. Además se incluía una sección para medir algunas competencias sociales y laborales, a través de autopercepciones.

\section{Gráfico 1}

Años de escolaridad por acierto en 5 o más preguntas del test preliminar

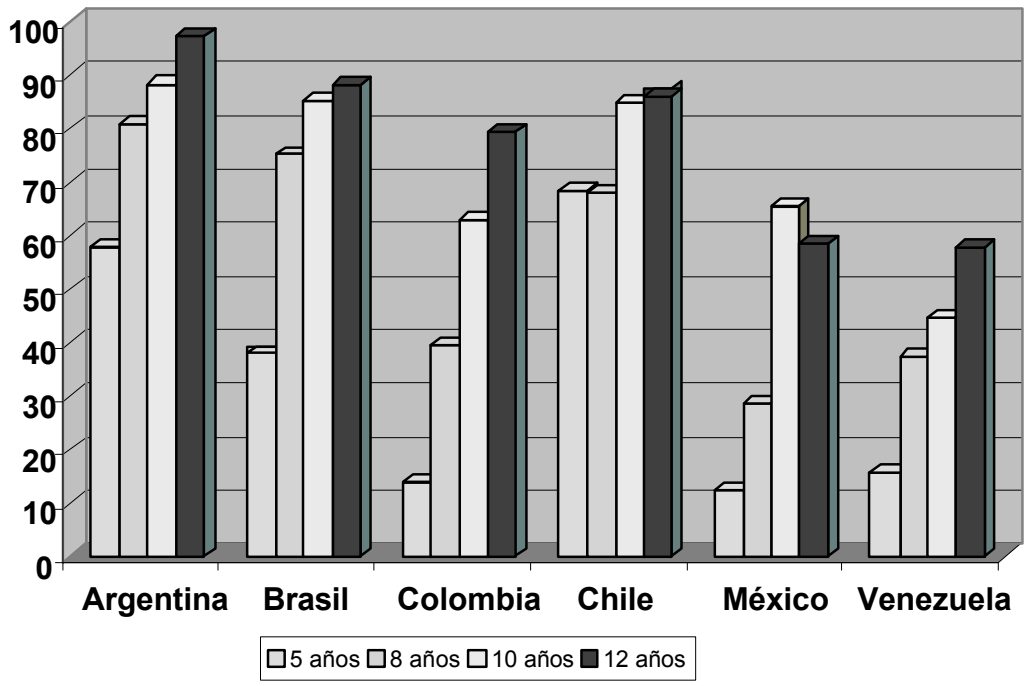

Educ. Soc., Campinas, vol. 23, n. 81, p. 71-89, dez. 2002 
La investigación se desarrolló en poblaciones entre 15 y 54 años de capitales o de ciudades importantes de Argentina (Gran Buenos Aires), Brasil (S. Paulo), Colombia (Bogotá), Chile (Gran Santiago) México (México, D.F., Monterrey y Mérida), Paraguay (Asunción y ciudades aledañas) y Venezuela (Gran Caracas).

El instrumento aplicado contenía una sección preliminar y otra principal, que se referían a las dominios de prosa, documentos (esquemas) y matemáticas o cálculo.

El test preliminar contenía sólo siete preguntas. Ese test servía de filtro para no cansar a las personas que difícilmente habrían contestado el test principal. El test preliminar ya arrojó resultados interesantes en la diferencia entre la calidad de los aprendizajes de personas adultas con algunos años de escolaridad en los diferentes países. El Gráfico no 1 muestra cómo con cinco años de escolaridad, tanto en Argentina como en Chile, más del 50\% contesta correctamente. No así en los demás países.

\section{Los niveles de alfabetismo}

Los niveles representan desarrollos diferentes de la habilidad, lo que se muestra en la resolución de tareas de diferente dificultad, relacionadas con la mayor o menor complejidad de la información y la exigencia, a partir de la tarea solicitada, de procesos más o menos complejos.

Con los resultados del test, la población se agrupó, según lo contestado en el test principal, en cuatro niveles de desempeño en cada uno de los dominios.

En prosa, las habilidades medidas son: identificar unidades informativas (palabras u oraciones), establecer relaciones entre ellas e inferir en textos de menor o mayor dificultad lingüística. La habilidad que aparece más difícil es la de inferir cuando se indaga acerca de causas o consecuencias. La más fácil, la de identificar palabras o frases con mínima o nula inferencia.

En documentos o esquemas, las habilidades medidas son las necesarias para procesar información que se presenta en forma de cuadros de menor o mayor complejidad, desde un anuncio de empleo hasta un aviso en que se incluye información no notoria que condiciona la resolución de la tarea.

En matemáticas, las habilidades medidas comprenden desde la habilidad necesaria para el cálculo de operaciones únicas (como adición), expresadas directamente en el enunciado de la tarea hasta las exigidas en el 
cálculo de operaciones secuenciales que deben inferirse de la información dada y cuya determinación se basa en conocimientos anteriores.

Los resultados de la investigación demostraron que en la mayoría de los países participantes son necesarios 12 o más años de escolaridad para alcanzar un nivel apropiado de competencias de lectura comprensiva de prosa, documentos y cálculo, que le permitiera insertarse adecuadamente en el medio laboral, lo que sucede fundamentalmente en el cuarto nivel.

Puso así de manifiesto el problema de la calidad de la enseñanza o de la calidad de los aprendizajes retenidos, provenientes de la educación básica o de la educación media. Aunque demostró diferencias entre los logros de los que habían cursado educación básica y media, la diferencia mayor, en cuanto a competencias, se produce entre la enseñanza escolar y la universitaria. Se pudo concluir que, en general, la escolaridad completa no garantiza un dominio real de las competencias de lectoescritura y matemáticas: los aprendizajes de la escuela (los que se retienen) no son suficientes para que las personas puedan enfrentar las exigencias de su medio o no corresponden a esas exigencias.

Como ejemplo, véase el Gráfico ${ }^{\circ}$ 2, referido a los niveles de prosa, documentos y matemáticas, relacionados con la variable escolaridad, en la muestra de Chile. Sólo los que tienen estudios superiores alcanzan en un $50 \%$ o más el cuarto nivel en prosa y matemáticas.

\section{Gráfico 2}

Años de escolaridad por niveles en Chile

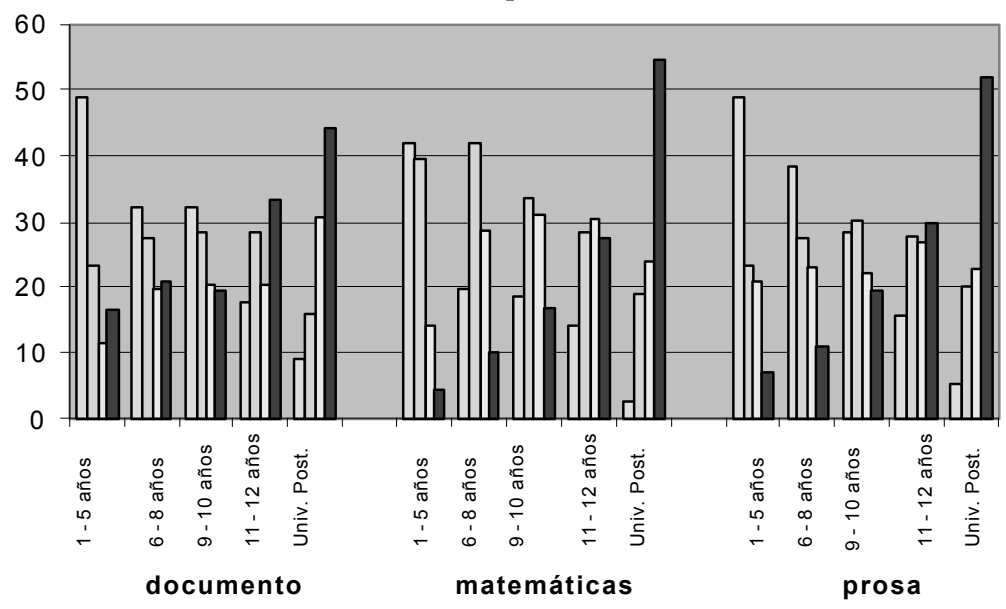

口Nivel $1 \square$ Nivel $2 \square$ Nivel $3 \square$ Nivel 4

Educ. Soc., Campinas, vol. 23, n. 81, p. 71-89, dez. 2002

Disponível em <http://www.cedes.unicamp.br> 
En la variable trabajo (alto, medio, bajo), se ve que los que tienen trabajo clasificado como bajo, es decir, ocupaciones en el área de industria, comercio, servicios, catalogadas como de nivel bajo (por ejemplo, obreros, vendedores ambulantes, porteros, empleadas domésticas), en un porcentaje alrededor de $60 \%$ pertenecen al primer y segundo nivel en todos los dominios y en todos los países de la investigación. ${ }^{4}$ Sin embargo, hay que hacer notar que entre los que tienen un trabajo clasificado como alto, entre un 25 y un $35 \%$ muestran un primer y segundo nivel de competencias. Habría que pensar que en la fuerza de trabajo aparentemente calificada también hay cierto porcentaje que no tiene competencias requeridas como básicas.

Por otra parte, se puede ver que hay porcentajes importantes de gente de todos los niveles de competencias que mantienen una inserción baja en el trabajo. Esto lleva a pensar que mucha gente tiene un nivel mayor de competencias que el nivel que el trabajo requiere.

En el siguiente gráfico $\left(\mathrm{n}^{\circ} 3\right)$, se puede ver la correlación entre niveles en prosa, documentos y matemáticas y la inserción laboral clasificada como baja, media y alta, en el mismo ejemplo anterior: Chile.

\section{Gráfico 3}

Inserción en el trabajo por niveles en los dominios de documentos, matemáticas y prosa en Chile

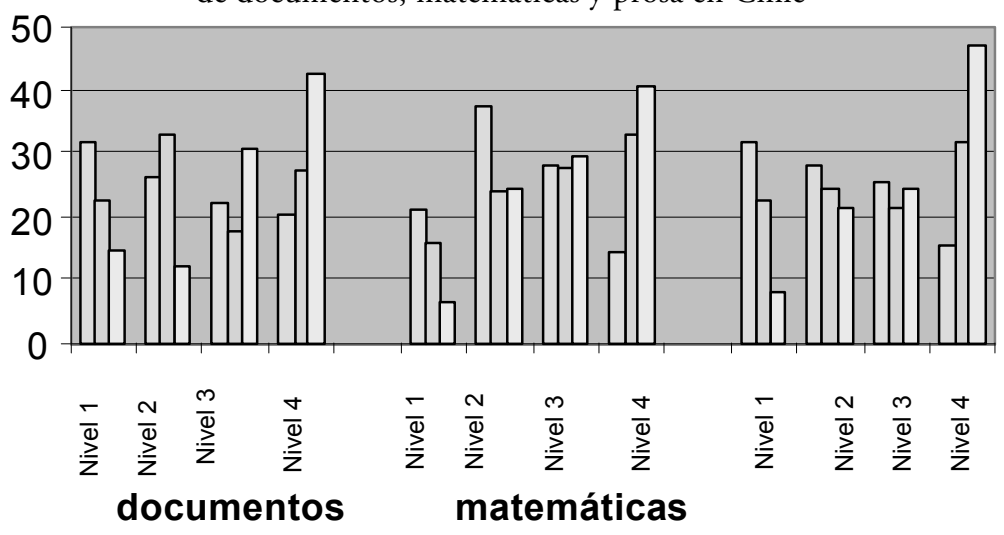

\section{$\square$ baja $\square$ media $\square$ alta}

Es importante señalar que también el uso de la lectura en el trabajo (medido a través de un índice) ${ }^{5}$ afecta significativamente en general los niveles de competencias en cada uno de los dominios, más que el uso de la lectura en la vida diaria. ${ }^{6}$ 
Explican también los niveles:

a) la escolaridad de los padres. Cuando hay diferencias, la escolaridad de la madre es todavía de mayor importancia que la del padre.

b) La zona de ubicación de la escuela primaria (urbana o rural) incide en algunos dominios en todos los países. El dominio más afectado es el de prosa. Esto se observa en Argentina, Brasil, Colombia y Chile, en los cuales el predominio del primer nivel entre los que estudiaron en escuelas rurales es muy fuerte, especialmente en Brasil y Colombia.

c) El tipo de escuela primaria (privada o pública) incide significativamente en los niveles de Brasil y México. Afecta en algunos dominios en Chile y Paraguay.

d) La zona de nacimiento (urbana, rural) es significativa en casi todas las investigaciones: en todos los dominios en Argentina y Brasil; sólo en prosa, en Colombia, Chile y México (en los demás dominios se observan tendencias en la misma dirección); en matemáticas en Paraguay.

\section{La medición de competencias sociales y laborales}

Algunas competencias laborales y sociales se midieron, como se dijo anteriormente, a través de autopercepciones. Se intentaba investigar si había alguna correlación entre ellas y los niveles de desempeño en alfabetismo. Las competencias medidas fueron la capacidad comunicativa, la capacidad de orientar, formar y organizar grupos, la capacidad de trabajar en grupo, la capacidad de seguir instrucciones, la capacidad de autonomía, la eficacia para solucionar problemas, la capacidad de alternativas, la capacidad manipulativa y la capacidad de vincularse al medio.

A pesar de las diferencias entre las investigaciones, se pueden ver líneas generales que indican caminos de posibles estudios posteriores. Las competencias que parecen más asociadas a los niveles en la mayoría de las investigaciones son la competencia comunicativa, la capacidad de seguir instrucciones y la capacidad de trabajo en equipo. También aparece vinculada a los niveles la conversación y discusión sobre la actualidad, que puede suponer mayor lectura o mayor esfuerzo por entender lo que pasa alrededor de cada persona. 
En cuanto a las competencias comunicativas, lo que más se destaca es la capacidad comunicativa que indica liderazgo, que incluye la capacidad de persuasión ("sé que puedo convencer a otros") y en algunos caso de orientación ("entiendo problemas de otros", "sirvo para aconsejar"), que aparece asociada a los niveles en algunos de los dominios en casi todos los países: en Brasil (prosa), Colombia (documentos y matemáticas), México (documentos y prosa), Argentina (prosa), Venezuela (documentos, matemáticas y prosa) y Chile (prosa y matemáticas).

\section{Principales hallazgos de la fase cualitativa}

El estudio, a través de la fase cualitativa, pudo comprobar que la escuela no asegura que perdure el gusto por la lectura y escritura. Mostró que el aprendizaje, adquirido por medios diferentes a la escuela, se relaciona con las estrategias para enfrentar el mundo letrado.

En contraparte, se pudo ver que la imagen de la escuela es muy importante en los recuerdos. En la mayoría de las personas que presentaron bajo rendimiento en el test, la recordaban con características negativas, muchas veces vinculadas a aspectos punitivos, en particular en lo que se refiere al aprendizaje inicial de la comunicación escrita. Mención especial merece el caso de las matemáticas: en casi la totalidad de los entrevistados fue la asignatura más difícil de aprender y estuvo ligada a amenazas y castigo incluso físico.

Por otra parte, hay que subrayar la importancia del maestro. En particular, en grupos de baja escolaridad y alto rendimiento en el test, la figura del maestro resaltó por la alta calidad afectiva con que se vinculó a alumnos y alumnas. Los que realizaron su etapa inicial en una escuela rural concibieron al maestro como una figura "insigne" de su comunidad, como un ejemplo en el actuar y el decir.

Frente a estas mediciones, hay que subrayar que, incluso frente a un texto escrito, la oralidad es el código más presente en nuestra cultura latinoamericana, en todos los grupos entrevistados, como lo mostró la fase cualitativa de la investigación. Sin embargo, mientras mejor se maneja el código escrito, mayor independencia hay frente al oral. No obstante, al código oral se recurre como estrategia para recabar más información acerca del texto escrito, acerca de las instrucciones que se imparten, de las tareas que se deben realizar. Este elemento no estuvo presente en el desarrollo del test, por tanto, la persona entrevistada no pudo sentirse como en una situación de la vida cotidiana. 
La investigación muestra la importancia del ambiente familiar, de la escolaridad del padre y de la madre en los logros de los jóvenes y adultos, influencia que seguramente se ejerce más fuertemente en los primeros aprendizajes. Al mismo tiempo, en la fase cualitativa se observó que las prácticas familiares relacionadas con la lectura marcaron de alguna manera la relación de las personas con el lenguaje escrito. En los hogares de las personas de niveles más altos de desempeño, existían materiales impresos, revistas, libros, no así en los hogares de las personas de bajo nivel de alfabetismo, para las cuales la escuela fue la principal referencia en la fase inicial de aprendizaje de la lectoescritura. Este aspecto debería considerarse como fundamental en los programas destinados a la educación básica de niños.

Ante los resultados de esta investigación, cabría preguntarse si las escasas competencias de lectura y escritura y cálculo, mostradas por un alto porcentaje de las poblaciones participantes, se relaciona sólo con los contenidos que se han entregado en la escuela, o si también influye la forma o metodología de enseñanza, es decir, si patrones rígidos metodológicos han limitado el desarrollo de estrategias y competencias; si han impedido que gran parte de la población pueda desenvolverse en forma flexible y esté en condiciones de aplicar en distintos ámbitos las habilidades desarrolladas. En el campo de la comprensión lectora, no se ha trabajado en la educación con formatos diferentes y con situaciones comunicativas distintas. En el de las matemáticas, no se han propuesto los variados problemas de las múltiples situaciones de la vida diaria. En ambos casos, no se estimuló el desarrollo de estrategias cognitivas de resolución de problemas.

\section{Confrontando las conclusiones del estudio con la situación actual}

Frente a la invasión de información y a la rapidez en que recibimos nuevos conocimientos, actualmente hay conciencia de que se necesita, en primer lugar, una sólida formación básica, que permita a las actuales generaciones "pensar" (es decir, analizar, sintetizar, inferir, interpretar...) adecuadamente una masa creciente de información y, en contraparte, dominar lenguajes diversos.

Se espera de la calificación intelectual que sea la base para los conocimientos específicos, que también son necesarios, y que además constituya la fuente principal de competencia que se prueba en la interacción y en las actividades concretas crecientemente complejas (Paiva, 1995). 
Enfocadas desde el trabajo, las competencias de base necesarias son las mismas que se exigen en la vida diaria. La alternancia de trabajos diferentes, o de períodos de desempleo, muestran la necesidad de que cada persona tenga una base sólida.

En cuanto a la base general, además de las competencias "duras" (comprensión de lectura en toda la gama de distinguir, relacionar, inferir; habilidades de matemáticas), se distinguen también competencias "blandas" (capacidad de trabajo en equipo, capacidad comunicativa, capacidad de autonomía) y competencias tecnológicas (uso de computadora).

Igualmente, en un contexto que cambia aceleradamente se necesita gran creatividad y flexibilidad para relacionar e imaginar situaciones nuevas, adaptarse y convivir con ellas, manejándolas.

Confrontando estas exigencias básicas con la realidad educativa de las personas adultas y los resultados de la investigación regional, se puede decir que actualmente las personas adultas, en su gran mayoría, no están capacitadas para enfrentarse a los desafíos de la sociedad del conocimiento y de la información. Esto traerá, sin duda, consecuencias graves en cuanto a mayor segmentación de las sociedades latinoamericanas. Sólo unos pocos tendrán la posibilidad de entrar realmente en el juego de la globalización y beneficiarse de ella. ${ }^{7}$

En contraparte, se puede constatar que en las políticas educativas, implementadas en nivel latinoamericano a partir de los años de 1990, la Educación de Personas Jóvenes y Adultas no ha tenido, en concreto, prioridad alguna. Sus acciones no se articularon con los procesos de reforma de los niveles de Educación Básica y Media ni contaron con recursos suficientes. Se redujeron sus equipos técnicos en los Ministerios de Educación, perdiendo influencia y notoriedad o desplazándose a otras tareas y modalidades (Rivero, 2002).

Se puede pensar que, mientras las reformas prioricen el espacio escolar y restrinjan en el discurso la educación a la educación escolar, la educación de adultos no se verá enfocada en la forma y medida necesarias y, por tanto, la educación en general no responderá a las necesidades del cambio de escenario del siglo XXI. Las reformas desarrolladas han sido pensadas de acuerdo a "una realidad social, política y cultural cada vez con menos probabilidades de permanencia y de proyección hacia el futuro" (Coll, 1999).

Sin embargo, el considerar que la educación en realidad es función y responsabilidad de toda la sociedad - lo que se venía 
afirmando ya desde hace unos 30 años -, y que la escuela, dentro de ella, es un agente importante, pero no el único ni el que tiene más peso en la educación, que lleva a diseñar políticas y estrategias diferentes que pueden responder mejor a los tiempos actuales y futuros.

$\mathrm{La}$ articulación entre los distintos actores (medios de comunicación, organismos de la sociedad civil y del Estado) que lleve a un consenso en la responsabilidad de enfrentar una nueva educación para los nuevos tiempos será básico para la configuración de estrategias y líneas de acción. De diversas maneras se ha denominado este enfoque en proyectos: "sociedad educativa", "comunidad de aprendizaje", "ciudad educadora". Sin embargo, todavía no se ha asumido de manera global.

En este contexto surge para la educación el desafío y la respuesta de la educación "permanente", una educación para toda la vida, para todas sus etapas y para todas los ámbitos en que la persona se mueve. $\mathrm{Y}$ en este ámbito cabe el diseño de una educación para personas jóvenes y adultas diferente, desescolarizada, que promueva fuertemente las destrezas culturales básicas, contextualizadas en los ámbitos de necesidades e intereses personales, sociales y laborales, permita continuar aprendiendo (educación superior, educación técnica) y sea capaz de presentar múltiples ofertas, de acuerdo a las necesidades de los diferentes públicos.

La tarea de incluir decididamente a la población adulta en las reformas debería constituir hoy una prioridad para todos los países de America Latina, si se quiere aumentar las competencias generales de la población que hoy día es activa, tanto para los niveles de competitividad del país como para disminuir los niveles de inequidad dentro de este.

Recebido e aprovado em outubro de 2002.

\section{Notas}

1. En términos de comparación, hay que señalar que como promedio en los países en desarrollo la diferencia de alfabetización entre hombres y mujeres en los mismos años alcanza a 22,1 puntos de porcentaje (1980) y a 17,2 (1995) y en los países desarrollados, a 2,6 (1980) y a 0,5 (1995).

2. Junto a Bolivia, Ecuador, El Salvador, Honduras, Paraguay, Perú y República Dominicana.

3. Más sobre esta investigación, conducida por la autora de este artículo, bajo el mandato de UNESCO/OREALC, en el libro publicado por UNESCO en abril de 2000: Alfabetismo funcional en siete paises de América Latina. 
4. Menos Paraguay, que tiene una muestra diferente: enfoca sólo a la población joven.

5. El uso de la lectura en el trabajo [alto (todos los días, algunas veces a la semana), medio (una vez a la semana), bajo (menos de una vez a la semana, nunca o casi nunca)] se trabajó a través de un índice, considerando para prosa preguntas como lectura o uso de: cartas o mensajes; informes, artículos o revistas; manuales o libros; hojas de instrucciones, recetas. Para lectura de documentos, se consideraron, para el índice: lectura o uso de diagramas o esquemas; formularios; interpretar gráficos, dibujos a escala, planos. Para el uso de matemáticas en el trabajo, el índice comprendió: lectura o uso de cuentas, facturas, planillas de cálculo, hojas de presupuesto; escritura de formularios o documentos como cuentas, facturas o presupuestos; medición o estimación del tamaño o peso de objetos; cálculo de precios, costos o presupuestos.

6. El uso de la lectura en la vida diaria de trabajó con un índice que comprendía (alto: diariamente o semanalmente; medio: todos los meses; bajo: algunas veces al año o nunca): ir a una biblioteca, leer diarios o revistas, leer libros.

7. "El principal peligro, en un mundo marcado por la interdependencia planetaria y la mundialización, es que se abra un abismo entre una minoría, capaz de moverse en ese mundo nuevo, y una mayoría impotente para influir en el destino colectivo." Informe Delors.

\section{Referencias Bibliograficas}

COLE, M. et al. The cultural context of learning andthinking: an exploration in experimental anthropology. In: SCRIBNER, S.; COLE, M. The psychology of literacy. Cambridge, Mass.: Harvard University, 1981.

COLL, C. Algunos desafios de la educación básica en el umbral de nuevo milenio. In: Seminario para Altos Directivos de las Administraciones Educativas de los países Iberoamericanos, 3., 1999. La Habana, 1999. p. 3.

COMISIÓN ECONOMICA PARA AMERICA LATINA Y EL CARIBE. Educación y conocimiento: eje de la transformación productiva con equidad. Santiago: CEPAL/OREALC, 1992. p. 41.

COMISIÓN ECONOMICA PARA AMERICA LATINA Y EL CARIBE. Panorama social de América Latina: 2000-2001.

DELORS, J. La educación encierra un tesoros. México, D.F.: UNESCO, 1996.

GAJARDO, M. Reformas educativas en América Latina: balance de una década. Santiago: Preal, 1999.

INFANTE, M.I. Alfabetización de jóvenes y adultos en América Latina: diagnóstico y perspectivas; ponencia presentada al Seminário Interna- 
cional sobre Educação e Escolarização de Jovens e Adultos. São Paulo: IBEAC, 1996.

INFANTE, M.I. Alfabetismo funcional en siete países de América Latina. Santiago: UNESCO/OREALC, 2000.

PAIVA, V. Inovação tecnológica e qualificação. Educação \& Sociedade, Campinas, n. 50, abr. 1995.

RIVERO, J. Bases curriculares en la educación con jóvenes y adultos latinoamericana: experiencias en cuatro países de la región; ponencia presentada al Seminario Internacional: Un marco curricular para la Educación de Adultos. Santiago: Ministerio de Educación de Chile, 2002.

SCHIEFELBEIN, E. et al. La enseñanza básica y el analfabetismo en América Latina y El Caribe: 1980-1987. Boletín Proyecto Principal de Educación en América Latina y El Caribe, Santiago, n. 20, p. 28-29, dic. 1989.

SOTOMAYOR, C. Stratégies de resolution de tâches de lecture chez des analphabètes fonctionnels chiliens. 1995. 273p. Tese (Doutorado) Université de Louvain-La-Neuve, Belgique.

TORRES, R. M. Itinerarios por la educación latinoamericana: cuaderno de viajes. Buenos Aires: Paidós, 2000. p. 21-

UNITED NATIONS EDUCATIONAL, SCIENTIFIC AND CULTURAL ORGANIZATION. Compendium of statistics on illiteracy. Paris: UNESCO, 1990.

UNITED NATIONS EDUCATIONAL, SICENTIFIC AND CULTURAL ORGANIZATION. Compendium of Statistics on Illiteracy. París: UNESCO, 1995.

UNITED NATIONS EDUCATIONAL, SCIENTIFIC AND CULTURAL ORGANIZATION. Situación educativa de América Latina y El Caribe: 1980- 1994. Santiago: UNESCO-SIRI, 1996. 